\title{
An Observation of Posterior Tibial Slope and Metaphysio- diaphyseal Angle in Indian Population
}

\author{
Vasudevan Thirunarayanan ${ }^{1}$, Dhurvas R Ramprasath ${ }^{2}$, Ramachandran Amarnath ${ }^{3}$, Velmani Arun ${ }^{4}$
}

\begin{abstract}
Introduction: The posterior inclination of the tibial plateau relative to the longitudinal axis of tibia is referred to as the posterior tibial slope (PTS). There is paucity of data regarding PTS in Indian population. Metaphysio-diaphyseal angle (MDA) is the angle between longitudinal axis of tibia and proximal tibial metaphysis, a new entity with a possible clinical significance. This study was performed to determine the mean PTS and mean MDA to study the correlation of PTS and MDA changes with osteoarthritic degeneration in Indian population and to assess the sensitivity and specificity of PTS and MDA in detecting osteoarthritis. A descriptive, cross-sectional study design was followed.

Materials and methods: A total of $173 \mathrm{X}$-rays with true PA and lateral views were examined from 121 individuals using standardized technique. Osteoarthritis was classified based on Ahlback grading system. Posterior tibial slope was defined as the angle formed by two lines in the lateral knee radiograph. Metaphysio-diaphyseal angle is a new entity defined in this study, formed between two lines-first line is the proximal anatomical axis of the tibia and the second is the axis of the proximal tibial metaphysis. All the observations and measurements of PTS and MDA were statistically analyzed using MedCalc software.

Results: There were 121 individuals in the study with 91 osteoarthritic knees and 82 normal knees. The mean PTS among normal group is $9.69^{\circ}$ [range $5-13^{\circ}$ with standard deviation (SD) 1.81] and among arthritic group is $14.05^{\circ}$ (range $10-24^{\circ}$ with SD 2.38). The mean MDA among normal group is $19.87^{\circ}$ (range $15-30^{\circ}$ with SD 2.70) and among arthritic group is $25.03^{\circ}$ (range $19-34^{\circ}$ with SD 3.05). There is a moderate correlation between PTS and MDA ( $r=0.64)$. Sensitivity and specificity in detecting osteoarthritis with PTS is $96.7 \%$ and $85.4 \%$ and by MDA is $90.1 \%$ and $84.7 \%$, respectively.

Conclusion: Our study finds that native PTS is similar to that of oriental population but higher than that of Caucasians. There is moderate linear correlation between PTS and MDA. They also serve as a marker in detecting osteoarthritis with good sensitivity and specificity.

Keywords: Knee, Metaphysio-diaphyseal angle, Osteoarthritis, Posterior tibial slope.

Journal of Orthopedics and Joint Surgery (2020): 10.5005/.jp-journals-10079-1023
\end{abstract}

\section{INTRODUCTION}

The coronal alignment of the knee has been evaluated extensively, but there are only few studies for the sagittal plane alignment. The posterior inclination of the tibial plateau in relation to its longitudinal axis in lateral view is the posterior tibial slope (PTS). Posterior tibial slope is an important factor that influences the sagittal alignment. ${ }^{1}$ Posterior tibial slope plays a very important role in the kinematics and biomechanics of knee joint. Studies in oriental population in respect to PTS have found different values from that of Caucasians. ${ }^{1,2}$ When PTS is high, the tibial shear force and anterior tibial translation also significantly rise. An increase in medial tibial slope may cause anterior cruciate ligament strain and injuries. ${ }^{3,4}$ Optimum intraoperative PTS is crucial in achieving good postoperative range of movements after total knee replacement (TKR).

Metaphysio-diaphyseal angle (MDA) is a recent radiological entity gaining clinical significance. It is the angle made between the longitudinal axis of tibia and the axis of proximal tibial metaphysis in a lateral view. The clinical importance of MDA is that it affects the mechanical axis in the sagittal plane by bringing the center of knee backward, thereby indirectly increasing the difference between anatomical axis obtained by Extramedullary jig and the mechanical axis of tibia while doing TKR. ${ }^{1}$ This study was performed to determine the mean PTS and mean MDA of normal knees and osteoarthritic knees in Indian population, to study the correlation of PTS and MDA changes in osteoarthritic degeneration, and to assess the sensitivity and specificity of PTS and MDA in detecting osteoarthritis.

\footnotetext{
1,3 Department of Orthopaedics, Government Kilpauk Medical College Hospital, Chennai, Tamil Nadu, India

${ }^{2}$ Department of Orthopaedic Surgery, Government Chengalpattu Medical College Hospital, Chengalpattu, Tamil Nadu, India

${ }^{4}$ Department of Orthopaedics, Government Dharmapuri Medical College Hospital, Dharmapuri, Tamil Nadu, India
}

Corresponding Author: Dhurvas R Ramprasath, Department of Orthopaedics, Government Chengalpet Medical College Hospital, Chenagalpet, Tamil Nadu, India, Phone: +91 9443464476, e-mail: dhurvasramprasath@gmail.com

How to cite this article: Thirunarayanan V, Ramprasath DR, Amarnath $\mathrm{R}$, et al. An Observation of Posterior Tibial Slope and Metaphysio-diaphyseal Angle in Indian Population. J Orth Joint Surg 2020;2(2):52-56.

Source of support: Nil

Conflict of interest: None

\section{Materials and Methods}

This study is a descriptive cross-sectional study done at our institution. Measurement of PTS and MDA on knees in patients of Indian Origin presenting to our OPD with arthritic knee pain were conducted during May to October 2016. The study group with normal knees was done with patients presenting to us with nonknee complaints after obtaining proper informed consent.

A total of $173 \mathrm{X}$-rays with lateral and PA views were examined from 121 patients using standardized technique. ${ }^{1}$ PA view was taken to grade the level of osteoarthritis per Ahlback grading system, which is classified as grade I (joint space narrowing $<3 \mathrm{~mm}$ ), grade II

(c) The Author(s). 2020 Open Access This article is distributed under the terms of the Creative Commons Attribution 4.0 International License (https://creativecommons. org/licenses/by-nc/4.0/), which permits unrestricted use, distribution, and non-commercial reproduction in any medium, provided you give appropriate credit to the original author(s) and the source, provide a link to the Creative Commons license, and indicate if changes were made. The Creative Commons Public Domain Dedication waiver (http://creativecommons.org/publicdomain/zero/1.0/) applies to the data made available in this article, unless otherwise stated. 

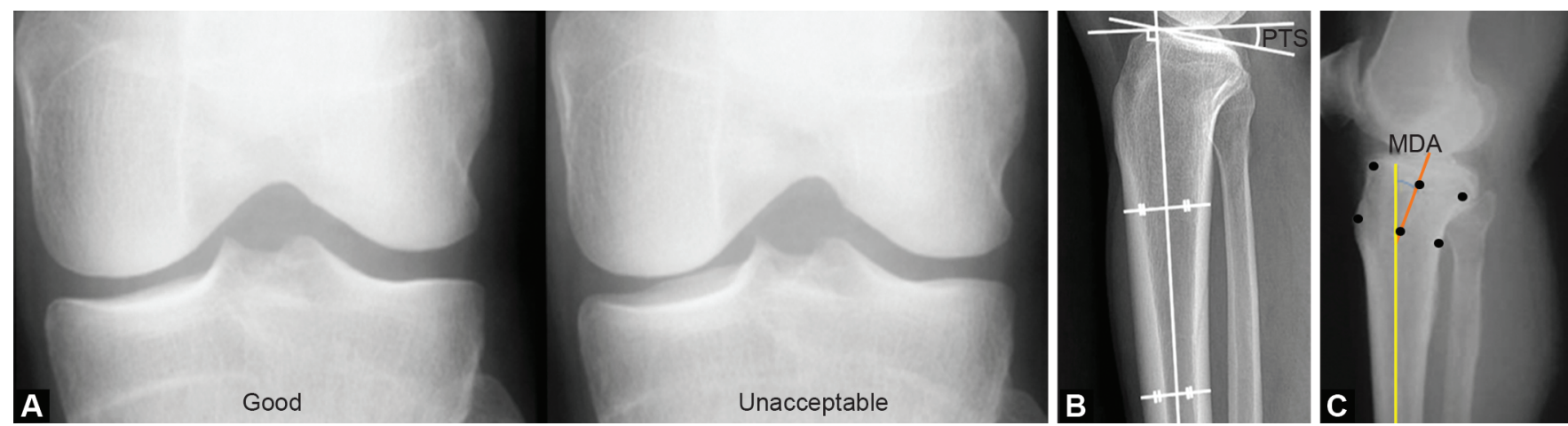

Figs 1 A to C: (A) PA view criteria; ${ }^{8}$ (B) X-ray lateral view of knee shows posterior tibial slope angle; ${ }^{1}$ (C) X-ray lateral view of knee shows metaphysiodiaphyseal angle ${ }^{1}$

(joint space obliteration), grade III (minor bone attrition 0-5 $\mathrm{mm}$ ), grade IV (moderate bone attrition $5-10 \mathrm{~mm}$ ), and grade $\mathrm{V}$ (severe bone attrition $>10 \mathrm{~mm}$ ). For PA view, the source is kept at a distance of $1.8 \mathrm{~m}$ with beam tilted $10^{\circ}$ caudally. ${ }^{1,2}$ In PA view, superposition of the posterior and anterior edges of the tibial plateau is required to accurately demonstrate the joint space. If the edge of the tibial plateau nearly touches or overlaps the distal femoral condyle on either knee, it becomes unacceptable (Fig. 1A). The radiologically acceptable lateral view of knee should have a clearly visible front of patella, tibial tuberosity, and top of patella with edges of all bone seen without bright light and front of femoral condyles overlie each other. ${ }^{1}$

Posterior tibial slope (Fig. 1B) was defined as the angle formed by two lines in the lateral knee radiograph. The first line was the line perpendicular to the anatomical axis of the tibia. The second line was formed by joining the most proximal points on the tibia plateau on the lateral radiograph as defined by Massin et al., ${ }^{5}$ avoiding osteophytes. Although there was no previous consensus on the ideal anatomical axis to measure PTS, the proximal anatomical axis, that is the line connecting midpoints of outer cortical diameter at 5 and $15 \mathrm{~cm}$ distal to the knee joint, is now recommended because it is most parallel to the sagittal mechanical axis. ${ }^{6}$ This axis was assumed to be the anatomical axis in our study also. Metaphysio-diaphyseal angle $^{1-5}$ is a new entity defined in this study. This angle is formed between two lines: first line is the proximal anatomical axis of the tibia and the second is the axis of the proximal tibial metaphysis. The axis of metaphysis (i.e., the second line) was drawn by defining two points each on anterior and posterior cortices of tibial metaphysis in a lateral film and joining the midpoints (Fig. 1C). Finally, the angle between these two lines (i.e., the MDA) was measured.

The study includes patients without any complaints in knee as normal group and patients with osteoarthritis as osteoarthritic group. The study excluded patients with fractures around knee and tibia, ligamentous injury of knee, 7,8 severe osteoporosis, tumors, Charcot's disease, osteolysis from any cause, and skeletally immature knees. All the observations and measurements of PTS and MDA are statistically analyzed using MedCalc software.

\section{Results}

The analysis of age distribution among our study group shows that the age-group 50-60 years is more affected with arthritis, with a mean age of 56.34 years (Fig. 2). The sex distribution in our study reveals there is a slightly increased prevalence of arthritis among female population. The PTS ranges from $5^{\circ}$ to $24^{\circ}$ in our study with mean PTS of $14.05^{\circ}$ [standard deviation (SD) 2.38] among

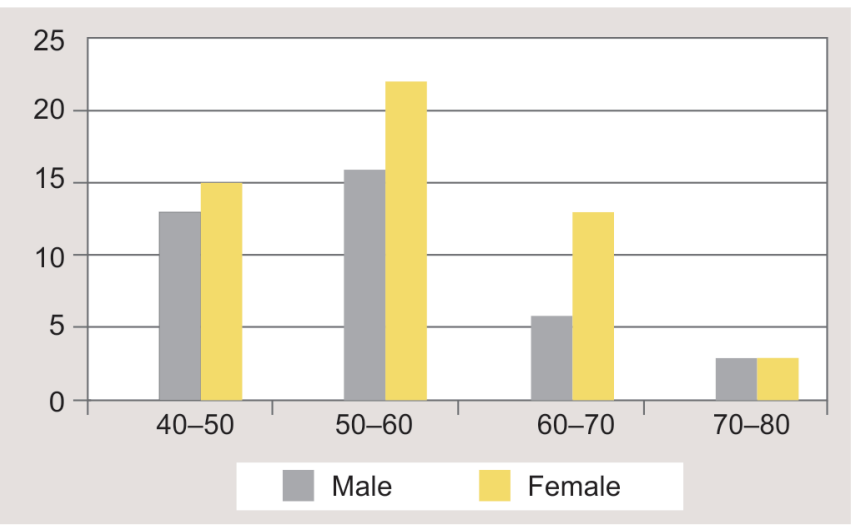

Fig. 2: Age wise distribution of osteoarthritis in male and female

osteoarthritic group and $9.69^{\circ}$ (SD 1.69) among normal group (Fig. 3A). The MDA of our study ranges from $15^{\circ}$ to $34^{\circ}$, with a mean MDA of $25.03^{\circ}$ (SD 3.053) among osteoarthritic and $19.87^{\circ}$ (SD 2.70) among normal group, respectively (Fig. 3B).

Receiver-operating characteristic (ROC) analysis provides tools to select possibly optimal models and to discard suboptimal ones independently from the cost context or the class distribution. It is related in a direct and natural way to cost-benefit analysis of diagnostic decision-making. The curve is created by plotting the true positive (sensitivity) results and false-positive (specificity) results at various threshold setting. The ROC curve for PTS gives sensitivity of $96.7 \%$ and specificity of $85.4 \%$ at a cutoff value of $11^{\circ}$ of PTS with $p$ value $<0.0001$ (Fig. 4A). The area under the curve (AUC) of PTS was found to be 0.975 which is statistically significant ( $p$ value $<0.0001$ ) (Table 1). The ROC curve for MDA gives a sensitivity of $90.1 \%$ and a specificity of $84.1 \%$ at a cutoff value of $22^{\circ}$ of MDA with $p$ value $<0.0001$ (Fig. 4B). The AUC of MDA was found to be 0.92 which is statistically significant ( $p$ value $<0.0001$ ) (Table 2 ). The PTS and MDA values are plotted in a graph and found to have a linear correlation with correlation coefficient $r=0.64$ that is a moderate correlation and statistically significant ( $p$ value $=0.0001$ ) (Fig. 5).

There were 121 patients in our study with 173 knee X-rays of which 91 were osteoarthritic knees and 82 were normal knees. The mean PTS among normal group is $9.69^{\circ}$ (range $5-13^{\circ}$ with SD 1.81) and among arthritic group is $14.05^{\circ}$ (range $10-24^{\circ}$ with SD 2.38). The mean MDA among normal group is $19.87^{\circ}$ (range $15-30^{\circ}$ with SD 2.70) and among arthritic group is $25.03^{\circ}$ (range $19-34^{\circ}$ with SD 3.053). There is a moderate correlation between PTS and MDA (correlation coefficient $r=0.64$ ). Regression analysis done with MDA 


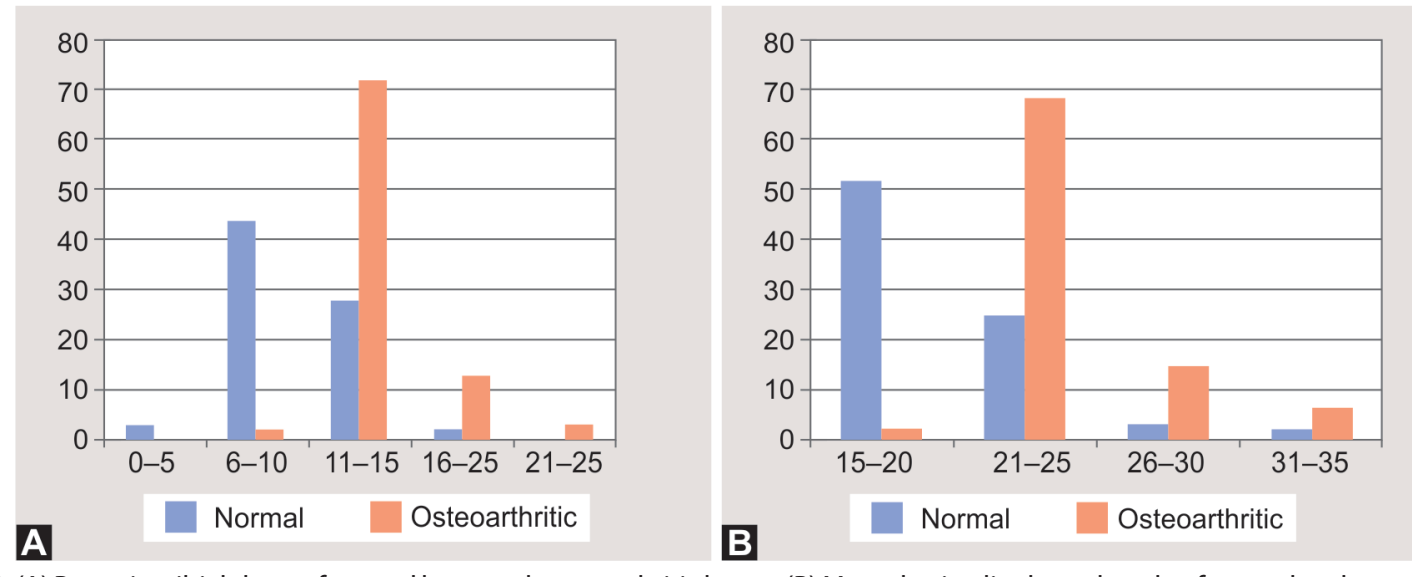

Figs 3A and B: (A) Posterior tibial slope of normal knee and osteoarthritic knees; (B) Metaphysio-diaphyseal angle of normal and osteoarthritic knees
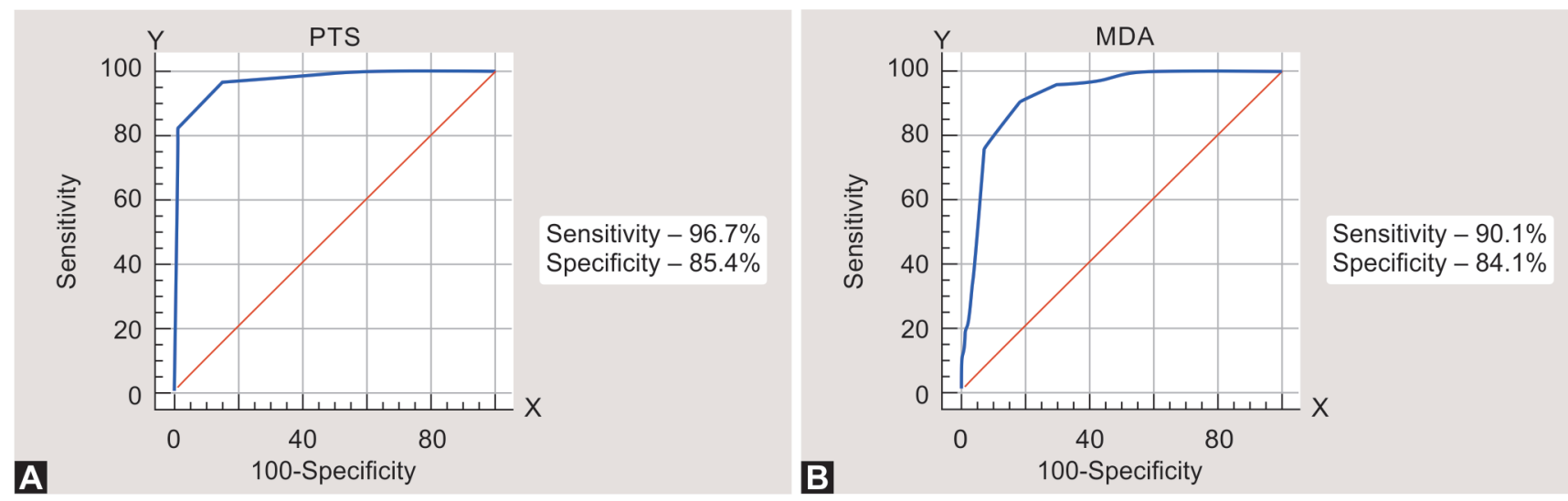

Figs 4A and B: (A) Receiver-operating characteristic (ROC) curve of posterior tibial slope for osteoarthritic group; (B) ROC curve of metaphysiodiaphyseal angle for osteoarthritic group

Table 1: AUC of PTS for osteoarthritic group

\begin{tabular}{ll}
\hline Area under the ROC curve (AUC) & 0.975 \\
Standard error & 0.00926 \\
$95 \%$ confidence interval & $0.939-0.993$ \\
$Z$ statistic & 51.274 \\
Significance level $p$ (area $=0.5)$ & $<0.0001$ \\
\hline
\end{tabular}

Table 2: AUC of MDA for osteoarthritic group

\begin{tabular}{ll}
\hline Area under the ROC curve(AUC) & 0.920 \\
Standard error & 0.0217 \\
$95 \%$ confidence interval & $0.869-0.956$ \\
$Z$ statistic & 19.358 \\
Significance level $p$ (area $=0.5)$ & $<0.0001$ \\
\hline
\end{tabular}

on $X$-axis and PTS on $Y$-axis gives an equation $y=0.4964+0.5060 x$ ( $p$ value $<0.0001$ ). Sensitivity and specificity for PTS are $96.7 \%$ and $85.4 \%$ and for MDA are $90.1 \%$ and $84.1 \%$, respectively.

\section{Discussion}

The PTS has been assessed using various methods ranging from direct cadaveric measurements to X-rays, computed tomography (CT), and magnetic resonance imaging (MRI) scans. The ideal way to measure the PTS by X-ray is against the anatomical axis of tibia. Both CT and MRI are useful in measuring the medial and lateral

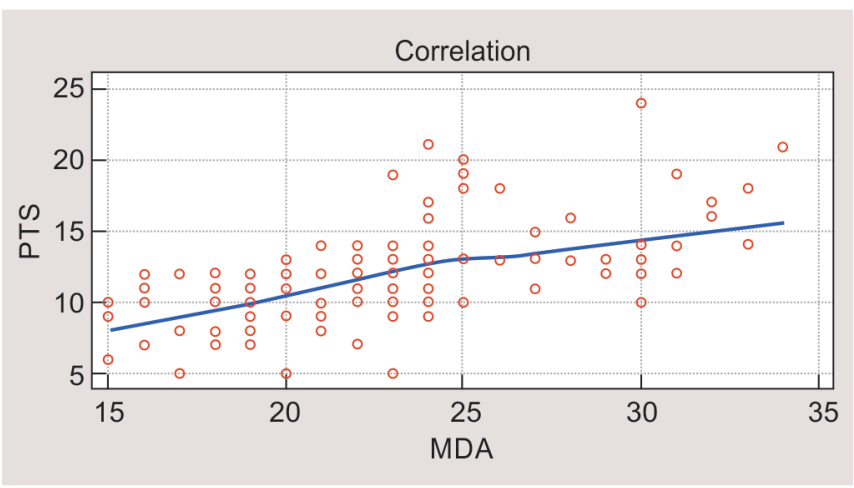

Fig. 5: Linear correlation of posterior tibial slope (PTS) and metaphysiodiaphyseal angle (MDA)

slopes separately. ${ }^{9,10}$ The age- and gender-specific PTS have not been fully mapped out for all populations. Among the few studied, it has been found to be different among different races. ${ }^{1,4-6,9,11,12}$

In our study, we have measured PTS of both normal and osteoarthritic knees with values of $9.69^{\circ}$ and $14.05^{\circ}$. Chiu et al. have also measured PTS of both normal and osteoarthritic knees with values of $10.8^{\circ}$ and $13.1^{\circ}$, respectively. Mohanthy et al. ${ }^{1}$ have measured PTS only in osteoarthritic knee with value of $11.64^{\circ}$, whereas all the other studies, such as Yoga et al., Khattak et al., Yoo et al., and Didia et al. have measured PTS for normal knees only and not for osteoarthritic knees (Table 3). 
An Observation of Posterior Tibial Slope and Metaphysio-diaphyseal Angle in Indian Population

Table 3: Comparison analysis of various studies done for posterior tibial slope (PTS) and metaphysio-diaphyseal angle (MDA)

\begin{tabular}{|c|c|c|c|c|c|c|c|c|}
\hline & \multicolumn{8}{|c|}{ Comparison analysis } \\
\hline & \multicolumn{2}{|c|}{ Normal knee } & \multicolumn{2}{|c|}{ Osteoarthritis } & \multicolumn{2}{|c|}{ PTS } & \multicolumn{2}{|c|}{$M D A$} \\
\hline & PTS & $M D A$ & PTS & $M D A$ & Sensitivity & Specificity & Sensitivity & Specificity \\
\hline Our study & 9.69 & 19.87 & 14.05 & 25.03 & $96.7 \%$ & $85.4 \%$ & $90.1 \%$ & $84.1 \%$ \\
\hline Mohanthy et al. & & & 11.64 & 23.76 & & & & \\
\hline Yoga et al. & 10.1 & & & & & & & \\
\hline Khattak et al. & 13.65 & & & & & & & \\
\hline Chiu et al. & 10.8 & & 13.1 & & & & & \\
\hline Yoo et al. & 10.6 & & & & & & & \\
\hline Didia et al. & 12.3 & & & & & & & \\
\hline Caucasians & 5.7 & & & & & & & \\
\hline
\end{tabular}

As per the comparison analysis shown earlier, the PTS in normal knees is found to be different in different studies. All the studies show that the PTS is higher in Asian population than Caucasians. Our study shows a mean of $9.69^{\circ}$ which is comparable to Yoga et al. of $10.1^{\circ}$. The highest for the Asians is $13.65^{\circ}$ which is shown by Khattak et al. ${ }^{2}$ The variations in PTS in Asian population as compared above maybe due to difference in reference axis and the methodology used in calculation. ${ }^{2,4,5,9,13}$ The increase in PTS with osteoarthritic degeneration has been shown in many studies. ${ }^{2,10,13}$ Our study also confirms that PTS increases with osteoarthritic degeneration. In our study, we have measured MDA in both normal and osteoarthritic knees with values of $19.87^{\circ}$ and $25.03^{\circ}$. Among the other studies, MDA has been measured only by Mohanthy et al. and that too only in arthritic knees with value of $23.76^{\circ}$.

In our study, we have not included post-TKR patients, so the actual clinical significance of PTS, with respect to post TKR, has not been done in our study. As per Yoga et al. ${ }^{9}$ who have studied the preoperative and postoperative TKR PTS, they have suggested that patients with higher preoperative PTS end up with higher postoperative PTS, which may increase the range of flexion, but these patients may not achieve full extension, which is not desirable. They also suggest that in patients with high preoperative PTS, when tibial cut is parallel to the surface, it exhibits $40 \%$ greater load-carrying capacity and $70 \%$ greater stiffness than paired tibiae cut perpendicular to long axis.

In this condition, to achieve full range of extension, an excess resection of proximal tibia needs to be taken. As per Bartel et al., ${ }^{11}$ when excess bone is resected in the proximal tibia, the stiffest and strongest cortical bone is removed, and the remaining weaker and less stiff cancellous bone stock often is inadequate to support the physiological loads of knee. So, in these patients with deficient bone in the proximal end of tibia, the mechanical support for the TKR component may be inadequate and may lead to loosening., ${ }^{9,11}$

According to Mohanthy et al., ${ }^{1}$ whenever MDA is higher, there will be an increased difference between the diaphyseal axis and mechanical axis in the sagittal plane. In TKA, tibial cuts are made using extramedullary jigs which is based on anterior tibial cortex. This will not compensate for any changes in MDA, and hence the aimed postoperative PTS may not be achieved. They have suggested a reference point of $20^{\circ}$ for MDA; above and below this $20^{\circ}$ value of MDA, the postoperative PTS gets altered. When MDA is less than $20^{\circ}$ and postoperative PTS of less than $3^{\circ}$ is aimed, then it might end up in a reverse tibial slope (anterior), whereas when it is greater than $20^{\circ}$ and a PTS of more than $5^{\circ}$ is aimed at, then this might result in a PTS much more than $5^{\circ}$. The anterior tibial slope might cause wear problems and the higher PTS of more than $5^{\circ}$ may result in loss of extension and component loosening. Mohanthy et al. also suggest that MDA has to be taken into consideration to achieve the desirable postoperative PTS and also to aim at a PTS of $3^{\circ}$ to $50^{\circ}$ which will be safe and avoid these possible errors.

The correlation between PTS and MDA in Mohanthy et al. study had a strong Pearson's coefficient correlation of 0.72 , which in our study was moderate correlation with $r=0.64$. We have further derived the regression analysis equation for PTS and MDA which is $y=0.4964+0.5060 x$ ( $p$ value $<0.0001)$, which helps us to calculate either PTS or MDA based on either one value.

Our study had certain limitations like the small sample size, and medial and lateral tibial slope vary in same patients. ${ }^{1,2,5,13}$ Computed tomography is the most accurate method in measuring PTS.,13-15 Gender-specific variation is not considered, and Clinical significance of PTS and MDA in TKR is not assessed

\section{Conclusion}

In our study, PTS in normal patients is higher than Caucasians and almost equal to that of oriental population. Our study finds that PTS and MDA show higher values in osteoarthritic group, and they have a moderate correlation between them. They also serve as a marker in detecting arthritis with good sensitivity and specificity. PTS is $96.7 \%$ sensitive in arthritic patients as a reliable marker with specificity of $85.4 \%$ and MDA is $90.1 \%$ sensitive with $84.1 \%$ specificity.

In our future course of study, we seek to continue this study with more samples to validate PTS and MDA as arthritic markers and its statistical significance in terms of sensitivity and specificity to study gender- and age-related changes of PTS and MDA and its statistical significance to study the slope changes with progression of arthritis and its statistical significance to further evaluate the significance of PTS and MDA with respect to TKR.

\section{References}

1. Mohanty SS, Rao NN, Dash KK, et al. Correlation of posterior tibial slope with metaphysio-diaphyseal angle in total knee arthroplasty: a radiological study. Indian J Orthop 2013;47(1):67. DOI: 10.4103/00195413.106910

2. Khattak MJ, Umer M, Davis ET, et al. Lower-limb alignment and posterior tibial slope in pakistanis: a radiographic study. J Orthop Surg 2010;18(1):22-25. DOI: 10.1177/230949901001800105.

3. Han HS, Chang CB, Seong SC, et al. Evaluation of anatomic references for tibial sagittal alignment in total knee arthroplasty. Knee Surg 
Sports Traumatol Arthrosc 2008;16(4):373-377. DOI: 10.1007/s00167008-0486-1.

4. Hashemi J, Chandrashekar N, Gill B, et al. The geometry of the tibial plateau and its influence on the biomechanics of the tibiofemoral joint. J Bone Joint Surg Am 2008;90(12):2724-2734. DOI: 10.2106/ JBJS.G.01358.

5. Massin P, Gournay A. Optimization of the posterior condylar offset, tibial slope, and condylar roll-back in total knee arthroplasty. J Arthroplasty 2006;21(6):889-896. DOI: 10.1016/j.arth.2005.10.019.

6. Yoo JH, Chang CB, Shin KS, et al. Anatomical references to assess the posterior tibial slope in total knee arthroplasty: a comparison of 5 anatomical axes. J Arthroplasty 2008;23(4):586-592. DOI: 10.1016/ j.arth.2007.05.006.

7. Shelburne KB, Kim HJ, Sterett WI, et al. Effect of posterior tibial slope on knee biomechanics during functional activity. J Orthop Res 2011;29(2):223-231. DOI: 10.1002/jor.21242.

8. Todd MS, Lalliss S, Garcia ES, et al. The relationship between posterior tibial slope and anterior cruciate ligament injuries. Am J Sports Med 2010;38(1):63-67. DOI: 10.1177/0363546509343198.

9. Yoga R, Sivapathasundaram N, Suresh C. Posterior slope of the tibia plateau in malaysian patients undergoing total knee replacement. Malays Orthop J 2009;10:4-21. DOI: 10.5704/MOJ.0911. 001.

10. Knee and Full Limb Radiography Operations Manual Version 1.0p May 2009.

11. Bartel DL, Burstein AH, Santavicca EA, et al. Performance of the tibial component in total knee replacement. J Bone Joint Surg Am 1982;64(7):1026-1033. DOI: 10.2106/00004623-19826407000009.

12. YauWP, Chiu KY,Tang WM, et al.Coronal bowing of the femur and tibia in Chinese: its incidence and effects on total knee arthroplasty planning J Orthop Surg 2007;15(1):32. DOI: 10.1177/230949900701500108.

13. Didia BC, Jaja BNR. Posterior slope of tibial plateau in adult nigerian subjects. Int J Morphol 2009;27(1):201-204. DOI: 10.4067/S071795022009000100034.

14. Brandon ML, Haynes PT, Bonamo JR, et al. The association between posterior-inferior tibial slope and anterior cruciate ligament insufficiency. Arthroscopy 2006;22(8):894-899. DOI: 10.1016/ j.arthro.2006.04.098.

15. Yue B, Varadarajan $\mathrm{KM}, \mathrm{Ai}$ S, et al. Differences of knee anthropometry between chinese and white men and women. J Arthroplasty 2011;26(1):124-130. DOI: 10.1016/j.arth.2009.11.020. 